\title{
Numerical Investigation to Quantify the Rate of Damage within Mortar Bituminous Materials: Modeling of Cracks Initiation and Propagation
}

\author{
Yassine El Haloui ${ }^{*}$, Aziz Idrissi Bougrine², Mohamed El Omari', El Khadir Lakhal' \\ Fateh-Fakhari Tehrani ${ }^{3}$, Joseph Absi ${ }^{4}$ \\ ${ }^{1}$ Cadi Ayyad University, Faculty of Sciences Semlalia, Laboratory of Automation, Environment and Transfer Processes ( LAEPT), \\ Boulevard Prince My Abdellah, P.B. 2390, 40000 Marrakech, Morocco \\ ${ }^{2}$ Cadi Ayyad University, Faculty of Sciences Semlalia, Department of Physics, \\ Boulevard Prince My Abdellah, P.B. 2390, 40000 Marrakech, Morocco \\ ${ }^{3}$ Conservatoire National des Arts et Métiers, 292 Rue Saint-Martin, 75003 Paris, France \\ 4 Université de Limoges, Institute of Research on Ceramics (IRCER), \\ UMR-CNRS 7315, 12 Rue Atlantis, 87068 Limoges, France \\ * Corresponding author, e-mail: yassine.elhaloui@edu.uca.ac.ma
}

Received: 03 December 2018, Accepted: 28 May 2019, Published online: 14 June 2019

\begin{abstract}
Asphalt concrete is highly used to construct pavement layers in the civil engineering field. It is defined as a complex medium composed of aggregates (inclusions), mortar (matrix) and air void. The mortar itself is a mixture of fillers, sand and bitumen. Furthermore, mortar is the phase that links the coarse aggregates. In general, fracture of asphalt concrete occurs within mortar or among aggregatemortar interface. Therefore, two types of fracture can be identified, i.e., adhesive and cohesive damages. The first type is occurred among the interface of aggregate-mortar. The second is taken place within the mortar. This paper presents numerical investigations of the damage initiation and stiffness degradation within the asphalt concrete matrix. Numerical simulations were carried out to investigate, firstly, how damage is initiated and developed, and then, to simulate how cracks can be initiated and propagated within this material. Cohesive finite elements method was adopted to simulate fracture. For adhesive damage, the model was represented by one rectangular aggregate that is linked to the asphalt concrete thanks to a thin layer of the mortar. For cohesive damage, the model was considered as a thick layer of the mortar in between two coarse aggregates. The applied loading was derived from the speed of traffic vehicle. A comparative analysis between four mortars was conducted. The effect of loading and the type of mortar on damage initiation and stiffness degradation will be shown. Moreover, the initiation and propagation of cracks as function of loading and stiffness modulus will be illustrated.
\end{abstract}

Keywords

damage, fracture, finite elements modelling, asphalt concrete, cohesive element, adhesive and cohesive damage

\section{Introduction}

Flexible pavement wearing course is usually constructed by using asphalt concrete materials that can be defined as a complex heterogeneous material composed of coarse aggregates, mortar, and a given percentage of air voids [1-3]. At the asphalt concrete scale (mesoscale), this material can be considered as a biphasic medium that comprises coarse aggregates and mortar. This latter is composed of fillers, sand, and bitumen $[4,5]$. The mechanical behavior of bituminous composites is viscoelastic. i.e., its mechanical properties depend on temperature and the load amplitude (frequency) [6-9].
In general, the overall fracture performance of asphalt concrete is dependent on the physical properties of each component, such as the moduli of aggregate and bitumen, cohesive and adhesive strength [10]. Damage occurs mostly along the aggregate-mortar interfaces and within the mortar itself [11]. However, aggregates are not considered to be damaged $[10,12]$. Damage along aggregatemortar interfaces is called adhesive damage. Though, damage within mortar is considered as cohesive damage. Adhesive damage is a result of loss bonding between aggregate outer surfaces and mortar at the region of contact. 
Whereas, cohesive damage is a result of stiffness degradation of matrix [13, 14]. Fig. 1 illustrates a fresh and damaged sample of asphalt concrete.

Various numerical methods can be used to model damage and fracture within materials. These methods are cohesive element and cohesive surface [15]. Cohesive element method could be used to simulate cohesive and adhesive damages. However, cohesive surface approach simulates adhesive damage. Many studies were carried out using cohesive surface and cohesive element methods. For example, cohesive element method was adopted by [13] to identify moisture damage at matrix. The adopted model was presented as one quarter of circular aggregate that is surrounded by a layer of a matrix. Cohesive surface method was used in the study of [14] to quantify moisture damage at the aggregate-mastic interface. The numerical model was represented as an aggregate that is coated by a layer of a matrix. The aggregate-matrix interface was simulated by using cohesive surface approach.

In the present study, numerical investigations, on damage state within mortar, will be presented. Cohesive element method is adopted to simulate damage initiation and propagation, and then, to model cracks initiation and propagation. In the literature, most researchers have applied a loading rate on their numerical models without any physical signification. In this paper, the displacement will be related to the speed of the vehicle to quantify damage rate.
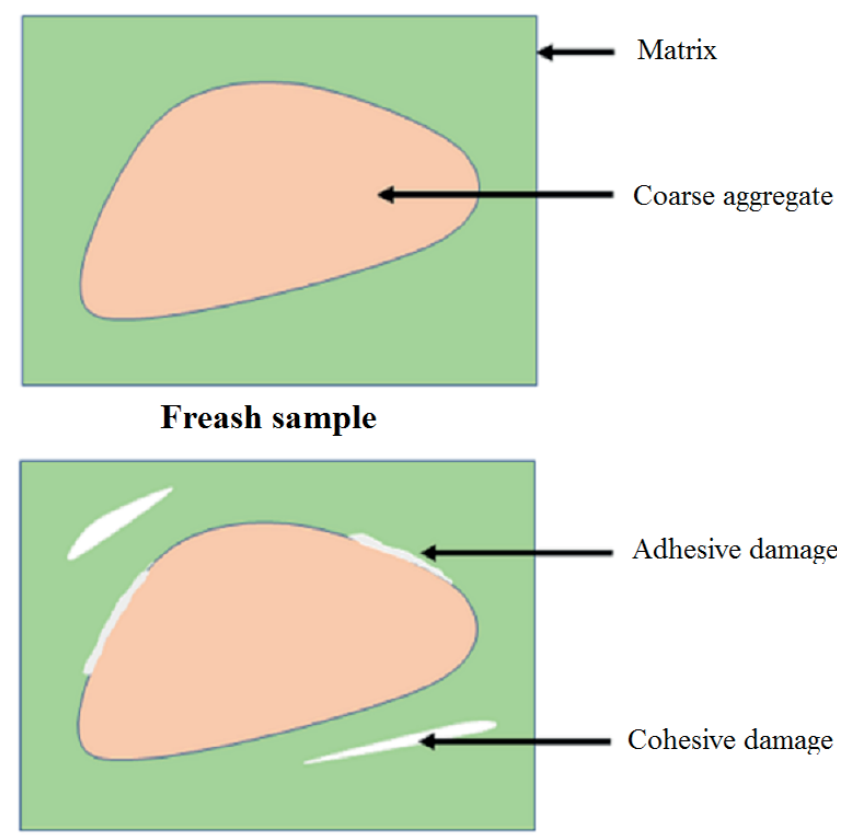

Damaged sample

Fig. 1 Illustration of adhesive and cohesive damage

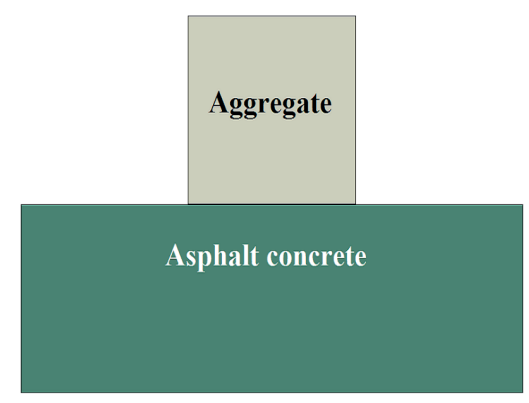

(a)

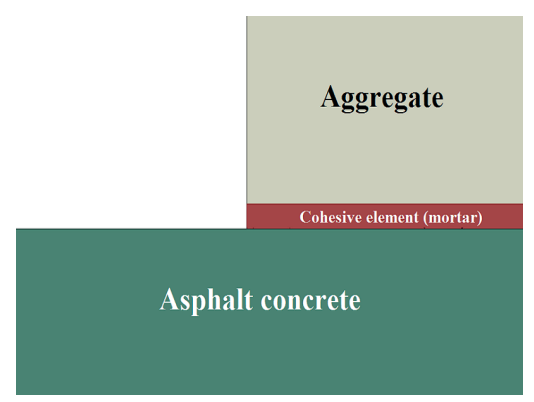

(b)

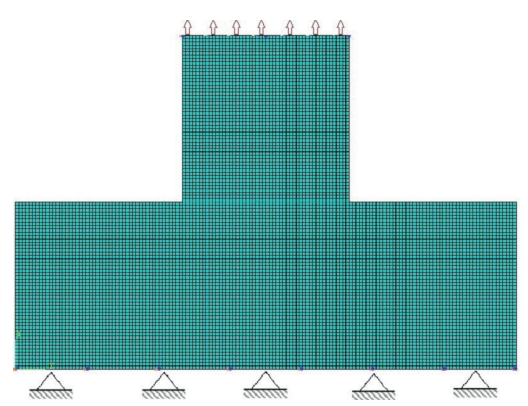

(c)

Fig. 2 Numerical model adopted in this study

The research paper aims basically at:

- Investigation on the effects of the speed and the fillers, used to formulate the four mortars, on damage initiation and stiffness degradation within mortar.

- Determination of the mortar type that provides the highest strength against traffic loading.

- Evaluating the impact of loading as well as stiffness modulus on the initiation and propagation of cracks.

\section{Numerical modeling steps}

ABAQUS/CAE version 2009 was used to perform the finite element simulation. The first adopted numerical model for this study is shown in Fig. 2. In general, aggregates have a complex shape that varies from circular to polygonal shapes. In this work, only two-dimensional rectangular aggregate is considered for the reason that the main objective is to investigate about damage initiation and stiffness degradation within mortar among the adhesive contact. 
Fig. 2(a) illustrates the global model; Fig. 2(b) shows the contact between aggregate and asphalt concrete that represented by the mortar. The adopted dimensions of aggregate and asphalt concrete are $10 \times 10 \mathrm{~mm}$ and $30 \times 10 \mathrm{~mm}$, respectively. The dimensions of mortar are $30 \times 0.5 \mathrm{~mm}$.

The adopted mesh and boundaries conditions applied to the numerical model are schematized in Fig. 2(c). The structured meshing method is adopted due to simplicity of the model with an overall mesh size equal to $0.1 \mathrm{~mm}$. In this study, the mortar is considered as cohesive material. Therefore, a 4-nodes two-dimensional cohesive element (COH2D4) is used to mesh the mortar. For aggregate and asphalt concrete, a 4-nodes bilinear plane stress quadrilateral (CPS4) is used as finite elements. The boundaries conditions are applied at the bottom surface of the model; both the vertical and horizontal displacements are constrained. A vertical displacement is applied at the top surface of aggregate as a function of the vehicle's speed.

\section{Damage law of cohesive element}

As discussed above, the cohesive element method can be used to simulate damage within materials. In this study, this method will be adopted to investigate the state of damage within the mortar. The cohesive law is composed of three parts. The first one is a linear elastic traction-separation behavior. The level of stress is less than that maximum stress (limit of the elastic domain). The second part is the initiation of damage. It is started when the stress exceeds the maximum one. The last part is the stiffness degradation that is represented by decreasing of the rigidity and the stress toward zero and thus the fracture of the cohesive element. The cohesive element law can be seen in Fig. 3. "A" is the point at which the damage will begin and "B" is the point at which fracture will be occurred. The elastic behavior can be written as described in the Eq. (1):

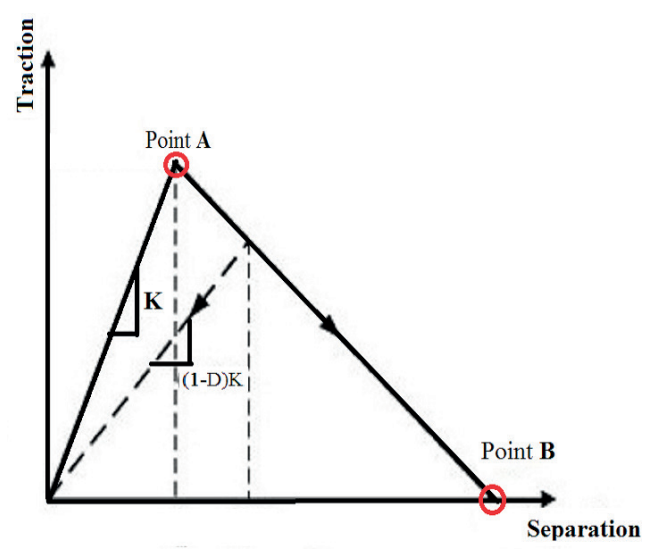

Fig. 3 Cohesive element mechanical behavior

$$
\left[\begin{array}{c}
\sigma_{n} \\
\tau_{s} \\
\tau_{t}
\end{array}\right]=\left[\begin{array}{ccc}
E_{n n} & 0 & 0 \\
0 & E_{s s} & 0 \\
0 & 0 & E_{t t}
\end{array}\right] \times\left[\begin{array}{c}
\varepsilon_{n} \\
\varepsilon_{s} \\
\varepsilon_{t}
\end{array}\right] .
$$

Where $\sigma_{n}, \tau_{s}$, and $\tau_{t}$ are the normal, first and second shear traction components, respectively. $E_{n n}, E_{s s}$, and $E_{t t}$ are the elastic moduli toward the normal, first, and second shear directions, respectively. $\varepsilon_{n}, \varepsilon_{s}$, and $\varepsilon_{t}$ are the normal, first, and second shear strain components, respectively.

The separation values are denoted by $\delta_{n}, \delta_{s}$, and $\delta_{t}$. The strain components can be defined as shown in Eq. (2):

$\varepsilon_{n}=\frac{\delta_{n}}{T} ; \varepsilon_{s}=\frac{\delta_{s}}{T} ; \varepsilon_{t}=\frac{\delta_{t}}{T}$,

where, $T$ is the initial thickness of the interface element.

The damage is assumed to begin when the maximum nominal stress criterion is reached. This criterion is given in Eq. (3):

$\operatorname{Max}\left\{\frac{\left\langle\sigma_{n}\right\rangle}{\sigma_{n}^{0}} ; \frac{\tau_{s}}{\tau_{s}^{0}} ; \frac{\tau_{t}}{\tau_{t}^{0}}\right\}=1$.

Where $\sigma_{n}^{0}, \tau_{s}^{0}$, and $\tau_{t}^{0}$ are the maximum stress toward the normal direction, first shear direction, and second shear direction, respectively. The Macauly brackets \langle\rangle indicate that a pure compressive stress state does not initiate the damage. In this paper, only two-dimensional model is considered. Thus, Eq. (3) becomes Eq. (4):

$\operatorname{Max}\left\{\frac{\left\langle\sigma_{n}\right\rangle}{\sigma_{n}^{0}} ; \frac{\tau_{s}}{\tau_{s}^{0}}\right\}=1$.

Stiffness degradation or damage evolution will start once the corresponding initiation criterion is reached (equations Eq. (3) in 3-D or Eq. (4) in 2-D). A scalar damage variable called $\mathrm{D}$ represents the overall damage in the cohesive element. The stress components during the damage evolution are detailed in Eq. (5), Eq. (6) and Eq. (7) as:

$\sigma_{n}=(1-D) \bar{\sigma}_{n}$,

$\tau_{s}=(1-D) \bar{\tau}_{s}$,

$\tau_{t}=(1-D) \bar{\tau}_{t}$,

where $\bar{\sigma}_{n}, \bar{\tau}_{s}$, and $\bar{\tau}_{t}$ are the stress components predicted by the elastic traction-separation behavior for the current strains without damage. 


\section{Description of materials and loading}

\subsection{Description of materials}

In general, aggregate is assumed to be as elastic material. Many studies identified the mechanical properties of aggregate as $[2,16]$. In this research, a Young's modulus of 48.3 GPa and Poisson's ratio of 0.20 were adopted to identify the aggregate in the numerical simulation.

Bituminous materials such as bitumen, mortar and asphalt concrete have a time-depend behavior. i.e., the mechanical properties are a function of temperature and frequency $[17,18]$. These materials can be behaved elastically at lower temperatures and viscoelastic at medium/higher temperatures. In this study, the experimental data were taken from the literature. The force ductility tests were conducted by [19] at low temperature. So, the mechanical behavior of mortar is assumed to be elastic. The experimental tests were carried out to investigate about the tensile cohesive property of mortar according to the European norm EN 13589. The temperature test was equal to $5^{\circ} \mathrm{C}$. Four types of mortar were formulated by using the same nature of bitumen (penetration grade of 80/100). Whereas, the skeleton of aggregates (fillers) was different; Limestone, Hydrated lime, Fly-ash and Diatomite fillers were used to formulate Limestone Asphalt Mortar (LAM), Hydrated lime Asphalt Mortar (HAM), Fly-ash Asphalt Mortar (FAM) and Diatomite Asphalt Mortar (DAM), respectively.

The maximum stresses of each type of mortar are used as input parameters in the numerical simulation. They can be determined from the force ductility values $F_{\text {Max }}$ using the equation $F=\sigma / e$. Where, $S$ is the contact surface which equal to $10 \mathrm{~mm}^{2}$. The shear modulus $G$ of each mortar is converted to the elastic modulus $E$ by using the following Eq. (8) [20]:

$$
E=2.5 G \text {. }
$$

The summary of damage model parameters is shown in Table 1. Asphalt concrete is defined by a Young's modulus of $30 \mathrm{GPa}$ and Poisson's ratio of 0.35 .

\subsection{Speed loading magnitudes}

The amplitude of loading was applied on the upper surface of aggregate (see Fig. 2 (c)). The magnitude of the displacements was taken from the literature. The influence of speed magnitude on the vertical displacement at the upper surface of pavement was shown in [21]. Based on this work, four speed magnitudes of 5, 20, 40, and $50 \mathrm{~m} / \mathrm{s}$ and their corresponding vertical displacement $0.832,0.860$, 0.880 , and $0.893 \mathrm{~mm}$, respectively, were used in this study.
Table 1 Inputs of the numerical simulation [19]

\begin{tabular}{lcccc}
\hline Mechanical properties & LAM & HAM & FAM & DAM \\
\hline$\left|G^{*}\right|(\mathrm{MPa})$ & 2.95 & 3.51 & 3.12 & 3.94 \\
$\left|E^{*}\right|(\mathrm{MPa})$ & 7.36 & 8.78 & 7.80 & 9.85 \\
$\sigma_{\mathrm{Max}}$ & 7.65 & 6.72 & 8.49 & 12.28 \\
\hline
\end{tabular}

Furthermore, two more displacement values of $0.508 \mathrm{~mm}$ and $1.200 \mathrm{~mm}$ were added to investigate about damage at speed magnitude lower than $5 \mathrm{~m} / \mathrm{s}$ and upper than $50 \mathrm{~m} / \mathrm{s}$. It should be noted that the speed magnitudes of $50 \mathrm{~m} / \mathrm{s}$ or upper than $50 \mathrm{~m} / \mathrm{s}$ are chosen in order to investigate about damage at higher loading conditions.

\section{Results and discussion}

\section{1 Damage initiation}

At this stage, an investigation of damage initiation will be shown. The damage is assumed to initiate when the maximum nominal stress criterion is reached (Eq. (4)). This criterion is defined and evaluated by a parameter called MAXSCRT (output of simulation). MAXSCRT varies between 0 (or $0 \%$ ) and 1.0 (or $100 \%$ ). When MAXSCRT is less than 1.0, it indicates that the damage has not begun yet within the mortar (cohesive elements). However, when MAXSCRT reaches its maximum value (1.0), the damage will be started.

The status of the mortar (the status of damage initiation criteria evaluated by MAXSCRT) can be seen in Fig. 4. From the cartography, it reveals that MAXSCRT value is higher at the lateral regions of the mortar. Moreover, these values decrease progressively toward the medium (center) region of the mortar. Consequently, damage initiation will begin first at the lateral regions and then will progress to the middle region.

Let's point out now on the effect of speed magnitude and the type of mineral aggregates on the parameter MAXSCRT (criterion of damage initiation). This effect is illustrated in Fig. 5.

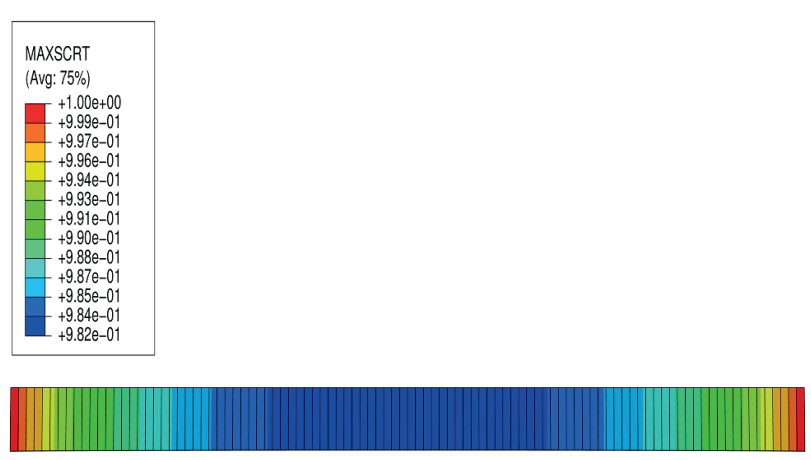

Fig. 4 MAXSCRT distribution of the mortar 


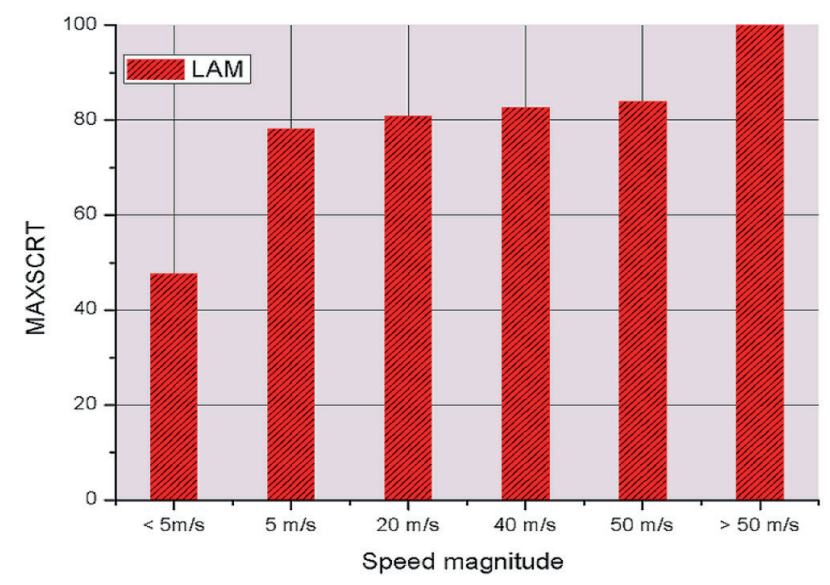

(a)

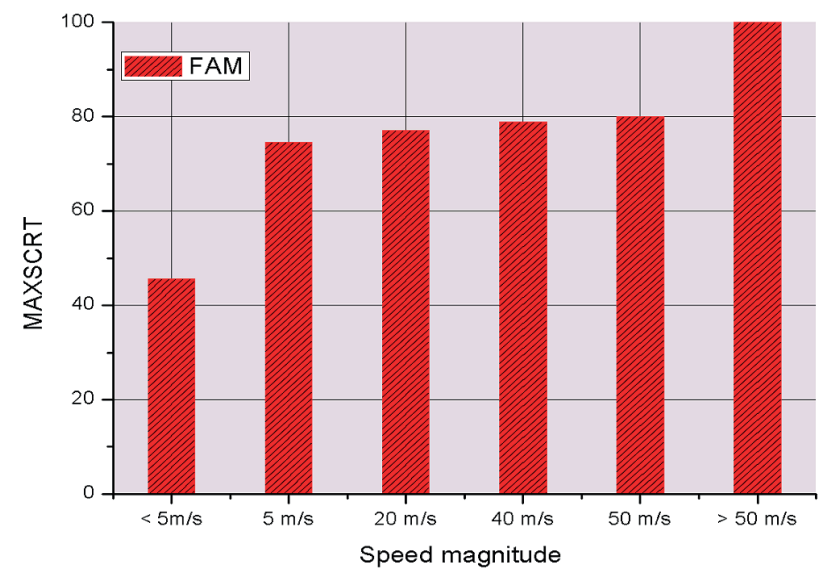

(c)

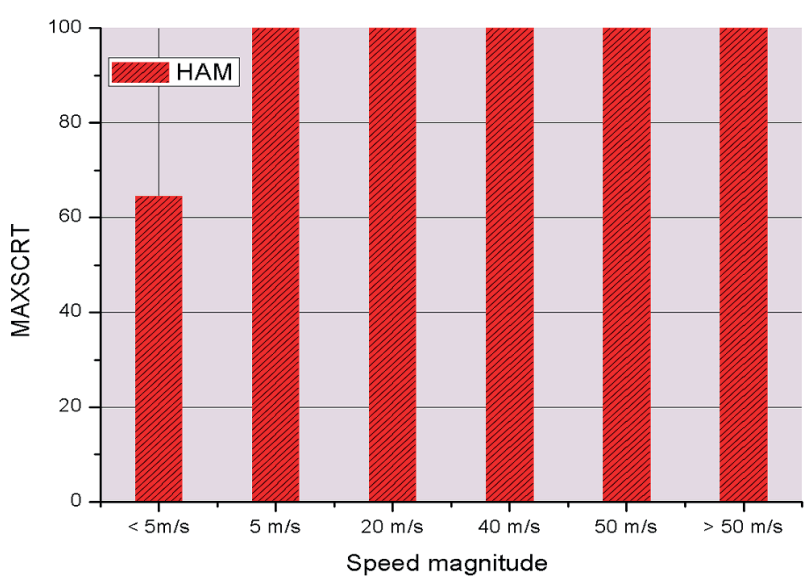

(b)

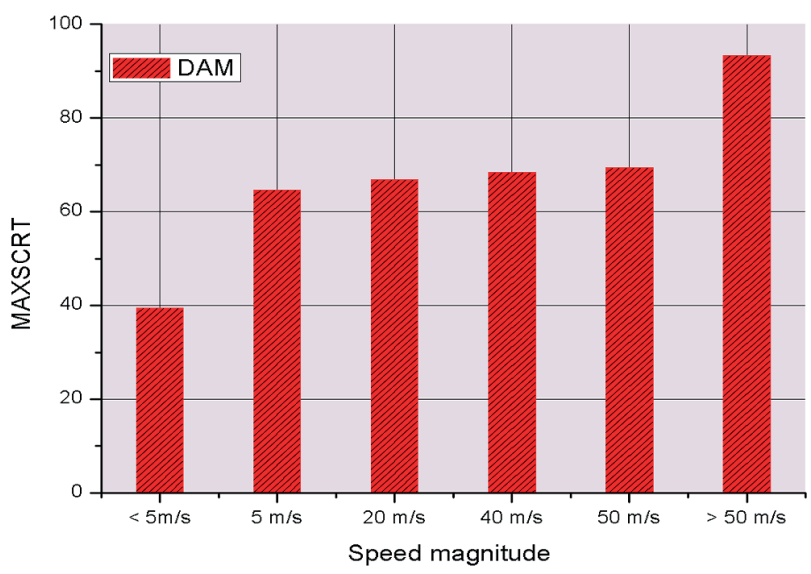

(d)

Fig. 5 MAXSCRT of the four mortars as a function of speed: (a) for LAM, (b) for HAM, (c) for FAM, and (d) for DAM

It can be seen that MAXSCRT increases as the speed increases. When the speed is lower than $5 \mathrm{~m} / \mathrm{s}$, MAXSCRT equal to 47.75, 64.56, 45.54, and 39.51\% for LAM, HAM, FAM and DAM, respectively. All the found values are less than $100 \%$. Therefore, damage has not been yet occurred under the considered speed. However, when the magnitude of speed achieves $5 \mathrm{~m} / \mathrm{s}$, it can be seen that the damage has been started in the case of HAM (Fig. 5(b)).

Besides, when the speed exceeds $50 \mathrm{~m} / \mathrm{s}$, on the one hand, the damage has been occurred within FAM (Fig. 5(c)) and LAM (Fig. 5(a)) cases. On the other hand, MAXSCRT value is still less than $100 \%$ for the case of DAM (Fig. 5(d)). Consequently, damage was initiated within all mortars except the one formulated by using Diatomite fillers (DAM).

The influence of the used fillers type to formulate the four mortars can also be revealed from the previous results in Fig. 5. This effect is compared when the speed magnitude is higher than $50 \mathrm{~m} / \mathrm{s}$. It is shown that Diatomite asphalt mor$\operatorname{tar}$ (DAM) shows the highest strength against damage initiation. In contrast, Hydrated lime Asphalt Mortar (HAM) shows the lowest strength against damage initiation.

\subsection{Stiffness degradation (damage evolution)}

As discussed above, MAXSCRT is the parameter that evaluates the initiation of damage. Let's now point out on the stiffness degradation that will be evaluated by using another parameter called SDEG (output of simulation). The stiffness degradation is assumed to start when the damage has already initiated. The scalar damage variable D (Eqs. (5-7)) is evaluated by SDEG. SDEG varies from $0(0 \%)$ to 1.0 $(100 \%)$. For example, the half of the stiffness is degraded if SDEG equal to 0.5 (50\%). A value of SDEG equal to 1.0 means that the stiffness is totally degraded and fracture will be occurred within the material.

The overall stiffness degradation of HAM under speed upper than $50 \mathrm{~m} / \mathrm{s}$ is presented in Fig. 6. It can be seen that SDEG equal to $54.06 \%$ at the lateral regions and it is less at the medium region of the mortar. As a result, the stiffness degradation rate is higher at the lateral regions than the medium one, and thus, the fracture within mortar will be occurred first at the lateral zones and then will progress to the middle zone. 

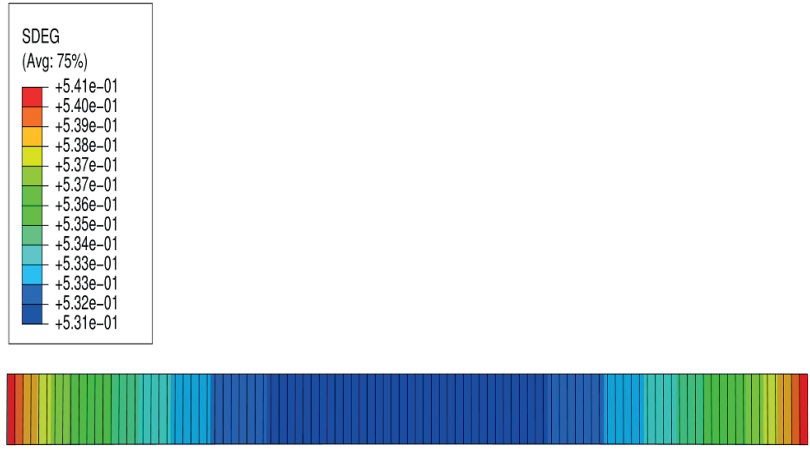

Fig. 6 SDEG distribution of the mortar

In this section, the effect of speed on stiffness degradation will be shown. The SDEG values of the matrixes under each speed magnitude are given in Fig. 7. It is clear that SDEG increases when the speed rises. In the case of HAM (Fig. 7(b)), SDEG increases from $0 \%$ at speed lower than $5 \mathrm{~m} / \mathrm{s}$ to $54.06 \%$ at speed upper than $50 \mathrm{~m} / \mathrm{s}$. However, there is no stiffness degradation in the case of DAM (SDEG $=0 \%$ Fig. 7(d)). This can be explained by the fact that damage has not begun yet in the case of DAM because MAXSCRT is still less than $100 \%$ (Fig. 5(d)).

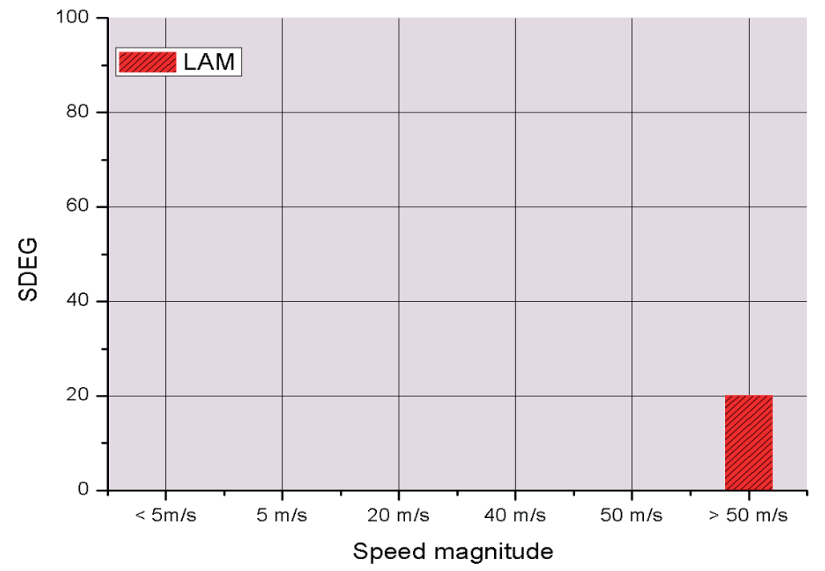

(a)

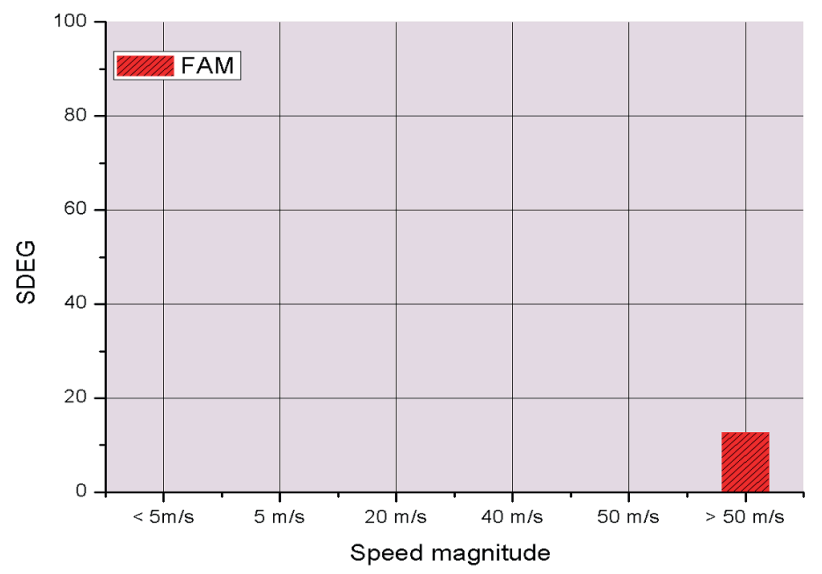

(c)
The degradation of the stiffness in the cases of LAM (Fig. 7(a)) and FAM (Fig. 7(c)) starts when the speed exceeds $50 \mathrm{~m} / \mathrm{s}$. LAM and FAM have a SDEG of $20.12 \%$ and $12.74 \%$, respectively.

At this level, the effect of fillers on stiffness degradation will be investigated. From the previous results, the stiffness degradation values are $20.12 \%, 54.06 \%, 12.74 \%$, and $0 \%$ of LAM, HAM, FAM, and DAM, respectively, at speed upper than $50 \mathrm{~m} / \mathrm{s}$. DAM shows the lowest value of SDEG; however, HAM shows the highest value of SDEG. As a result, the mortar formulated by using Diatomite fillers has the lowest stiffness degradation. However, the mortar formulated by using hydrated lime shows the highest stiffness degradation under the same loading condition.

\section{3 Modeling of cohesive fracture within mortar}

Cohesive damage is the type of fracture that can be occurred within the mortar and not at the interface aggregate-mortar. This part is dedicated to modeling the initiation and propagation of cracks. The adopted numerical model is given in Fig. 8.

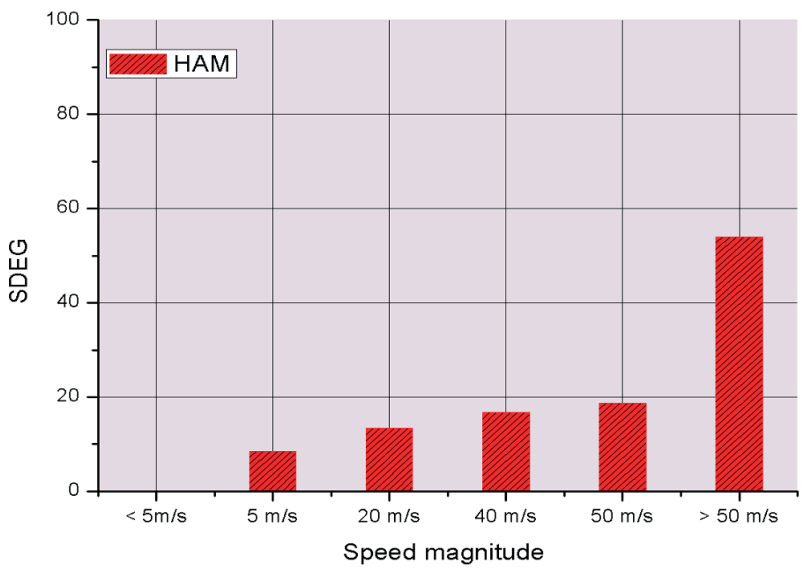

(b)

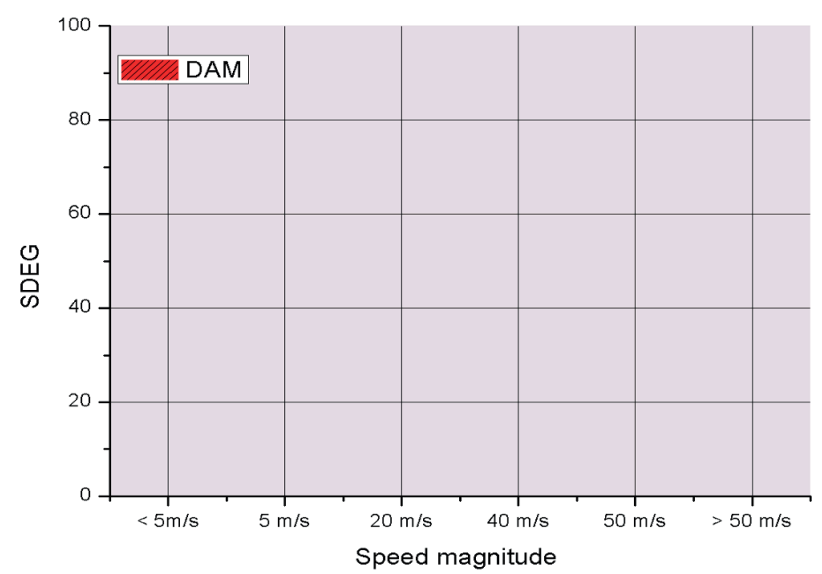

(d)

Fig. 7 SDEG of the four mortars as a function of speed: (a) for LAM, (b) for HAM, (c) for FAM, and (d) for DAM 


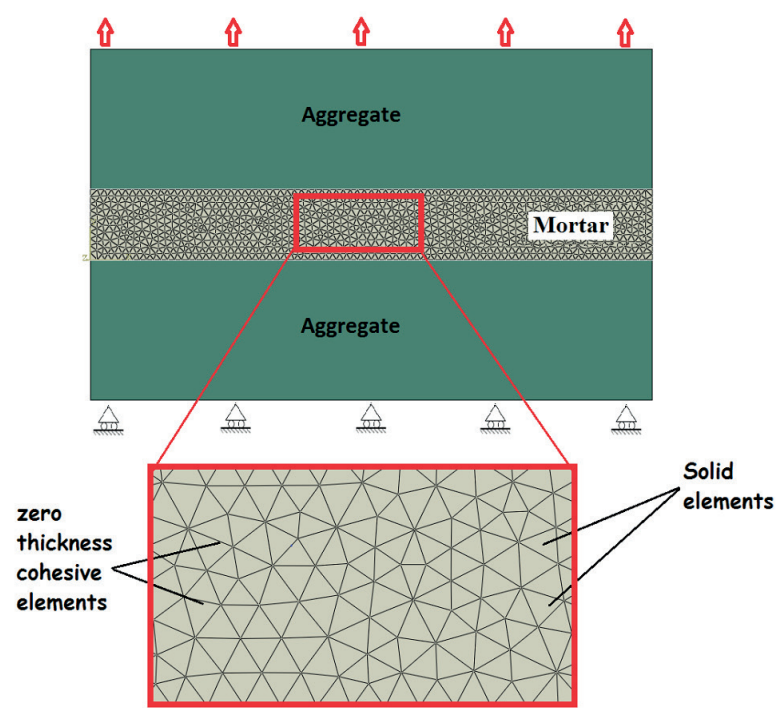

Fig. 8 Description of the numerical model

Zero thicknesses cohesive elements were inserted between the solid elements (finite elements) of mortar. The method of insertion can be summarized by two steps. The first one is to mesh the mortar through using ABAQUS software by adopting triangular finite elements. The second step is the modification of the initial mesh as follows: Each common node links, of at least two solid elements, will be duplicated (the number of duplication equal to that of solid elements linked by the node). After that, a cohesive element with zero thicknesses will insert within solid elements (the nodes of the cohesive elements are the duplicated nodes). More information of the method of insertion can be found in [22]

The dimensions of the numerical model are aggregates $40 \times 10 \mathrm{~mm}$ and mortar $40 \times 5 \mathrm{~mm}$. A static step simulation is considered with a total time equal to $1 \mathrm{~s}$. The applied displacement is increasing from 0 to $5 \mathrm{~mm}$ on the top surface of aggregate.

\subsubsection{Effect of loading}

Fig. 9 shows the cracks initiation and propagation within the mortar during the time of the step as the loading increases. It is important to mention that the scale of mortar is increased in order to visualize the crack paths. Figs. 9(a-d) illustrate the status of mortar at the step times of $0,0.2,0.3$, and $1 \mathrm{~s}$, respectively. It reveals that two cracks have been initiated and then propagated as the loading increases. The first crack has been started at the top left location of the mortar. After that it is horizontally propagated from the left to the right. Nevertheless, the second crack has been initiated at the bottom right location and then horizontally propagated from the right to the left for about $10 \mathrm{~mm}$.

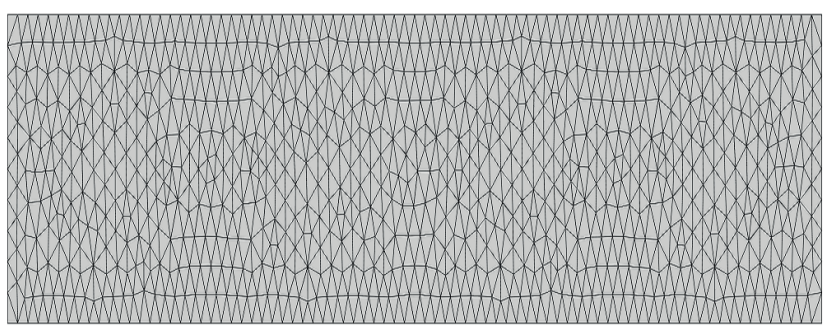

(a)

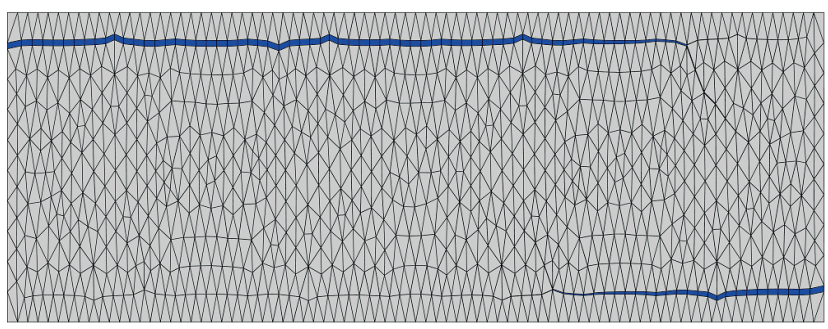

(b)

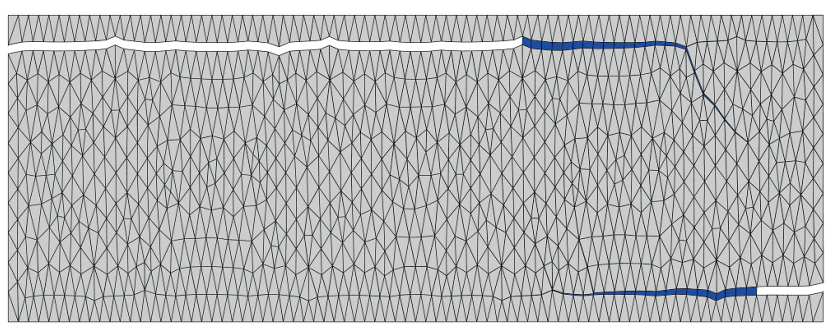

(c)

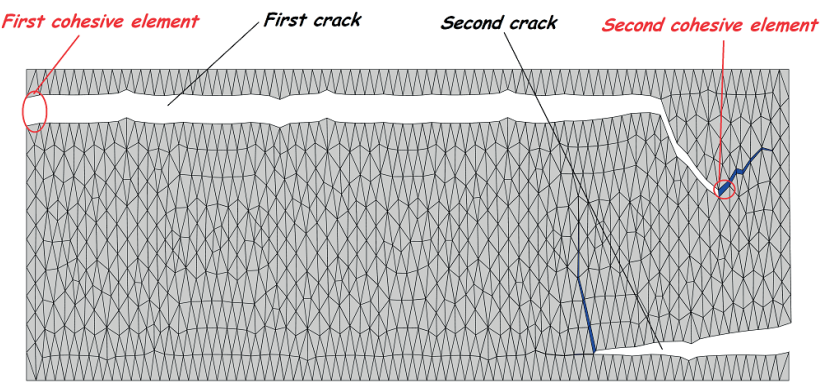

(d)

Fig. 9 Initiation and propagation of cracks: $t=0 \mathrm{~s}$ for (a), $t=0.2 \mathrm{~s}$ for (b), $t=0.3 \mathrm{~s}$ for (c), and $t=1 \mathrm{~s}$ for (d)

\subsubsection{Effect of the Young's modulus}

In general, fracture depends on several parameters such as the mechanical properties of mortar as the stiffness modulus (Young's modulus) [23]. The Young's modulus $E$ adopted in the aforementioned case was 18.2 GPa. To examine how $E$ affects the behavior of fracture behavior, two more values of $\mathrm{E}$ have been selected as follows: $50 \%$ of $E$ $(9.1 \mathrm{GPa})$ and $150 \%$ of E (27.3 GPa). Fig. 10 shows the crack paths within mortar. Figs. 10(a) and 10(b) illustrate the paths of cracks in the case of $E=9.1 \mathrm{GPa}$ and $E=27.3 \mathrm{GPa}$, respectively. It can be seen that only one crack has been appeared and the mortar has been divided into two parts in the case of $E=9.1 \mathrm{GPa}$. However, when the stiffness 


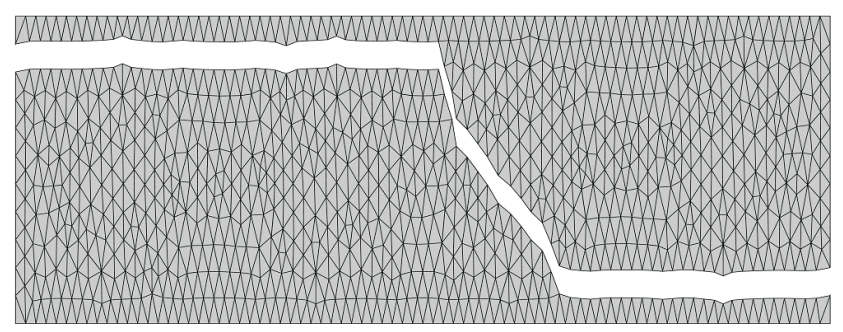

(a)

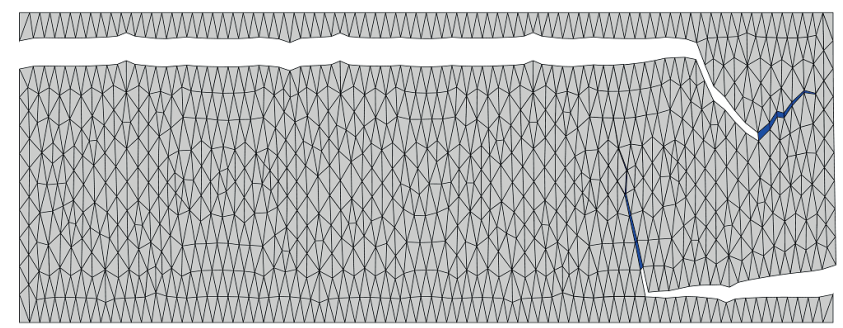

(b)

Fig. 10 Paths of cracks within mortar: $E=9.1 \mathrm{GPa}$ for (a) and $E=27.3$ GPa for (b)

equal to $27.3 \mathrm{GPa}$, the crack paths are identical to the those found when $E=18.2 \mathrm{GPa}$. The only difference between those paths is that in the case of the second crack (crack at the bottom right location), there is one more cohesive element that is separated in the case of stiffness equal to 27.3 GPa (see Figs. 9(d) and 10(b)).

\section{References}

[1] El Haloui, Y., Tehrani, F. F., Absi, J., Courreges, F., El Omari, M., Allou, F., Petit, C. "Micromechanical Behaviour of Asphalt Concrete Based on X-ray Computed Tomography Images and Random Generation of Heterogeneous Aggregates Skeleton", IOP Conference Series: Materials Science and Engineering, 416, 2018. https://doi.org/10.1088/1757-899X/416/1/012054

[2] EL Haloui, Y., Hajikarimi, P., Tehrani, F. F., Absi, J., El Omari, M., Petit, C. "Micromechanical modeling of the interfacial zone in hot mix asphalt through use of a heterogeneous numerical method", European Journal of Environmental and Civil Engineering, 2018. https://doi.org/10.1080/19648189.2018.1492462

[3] Lublóy, É., Ambrus, D., Kapitány, K., Barsi, Á. "Air Void Distribution of Asphalts Determined by Computed Tomography", Periodica Polytechnica Civil Engineering, 59(4), pp. 503-510, 2015. https://doi.org/10.3311/PPci.7608

[4] Tehrani, F. F., Absi, J., Allou, F., Petit, C. "Investigation into the impact of the use of 2D/3D digital models on the numerical calculation of the bituminous composites' complex modulus", Computational Materials Science, 79, pp. 377-389, 2013. https://doi.org/10.1016/j.commatsci.2013.05.054

[5] Tehrani, F. F., Absi, J., Allou, F., Petit, C. "Heterogeneous numerical modeling of asphalt concrete through use of a biphasic approach: Porous matrix/inclusions", Computational Materials Science, 69, pp. 186-196, 2013.

https://doi.org/10.1016/j.commatsci.2012.11.041

\section{Conclusions}

Based on the above results, the following conclusions are drawn:

- The damage initiation criterion in the mortar is a function of the speed magnitude. When the speed increases, the damage initiation criterion also increases.

- The DAM shows the highest strength against the damage initiation. However, the HAM shows the lowest strength against the damage initiation over the same loading condition.

- The overall stiffness degradation (SDEG) for each matrix is a function of the speed magnitude. When the speed increases, SDEG also increases.

- The DAM shows the lowest stiffness degradation, while the HAM shows the highest stiffness degradation against the same loading condition.

- Cracks were initiated and then propagated within the mortar as the applied rate of loading is increasing.

- The value stiffness modulus can affect the cracking paths within the mortar.

[6] Di Benedetto, H., Olard, F., Sauzéat, C., Delaporte, B. "Linear viscoelastic behaviour of bituminous materials: From binders to mixes", Road Materials and Pavement Design, 5(1), pp. 163-202, 2004. https://doi.org/10.1080/14680629.2004.9689992

[7] Si Bachir, D., Dekhli, S., Mokhtar, K. A. "Rheological Evaluation of Ageing Properties of SEBS Polymer Modified Bitumens", Periodica Polytechnica Civil Engineering, 60(3), pp. 397-404, 2016. https://doi.org/10.3311/PPci.7853

[8] Tóth, Cs., Ureczky, J. "Determination of master curves for asphalt mixtures by means of IT-CY tests", Periodica Polytechnica Civil Engineering, 54(2), pp. 137-142, 2010.

https://doi.org/10.3311/pp.ci.2010-2.09

[9] El Haloui, Y., Tehrani, F. F., Absi, J., Courreges, F., El Omari, M., Allou, F., Petit, C. "Modelling of asphalt mixes based on X-ray computed tomography and random heterogeneous generation", International Journal of Pavement Engineering, 2018. https://doi.org/10.1080/10298436.2018.1559316

[10] Yin, A., Yang, X., Yang, S., Jiang, W. "Multiscale fracture simulation of three-point bending asphalt mixture beam considering material heterogeneity", Engineering Fracture Mechanics, 78(12), pp. 2414-2428, 2011. https://doi.org/10.1016/j.engfracmech.2011.06.001

[11] Kim, H., Buttlar, W. G. "Discrete fracture modeling of asphalt concrete", International Journal of Solids and Structures, 46(13), pp. 2593-2604, 2009. https://doi.org/10.1016/j.ijsolstr.2009.02.006 
[12] Teixeira, J. E. S. L., Kim, Y. R., Souza, F. V., Allen, D. H., Little, D. N. "Multiscale Model for Asphalt Mixtures Subjected to Cracking and Viscoelastic Deformation", Transportation Research Record: Journal of the Transportation Research Board, 2447(1), pp. 136-145, 2014. https://doi.org/10.3141/2447-15

[13] Hossain, M. I., Tarefder, R. A. "Identifying damage in asphalt matrix materials surrounding an aggregate particle", Construction and Building Materials, 49, pp. 536-546, 2013. https://doi.org/10.1016/j.conbuildmat.2013.08.083

[14] Hossain, M. I., Tarefder, R. A. "Quantifying moisture damage at mastic-aggregate interface", International Journal of Pavement Engineering, 15(2), pp. 174-189, 2014. https://doi.org/10.1080/10298436.2013.812212

[15] Song, S. H., Paulino, G. H., Buttlar, W. G. "A bilinear cohesive zone model tailored for fracture of asphalt concrete considering viscoelastic bulk material", Engineering Fracture Mechanics, 73(18), pp. 2829-2848, 2006. https://doi.org/10.1016/j.engfracmech.2006.04.030

[16] Tehrani, F. F., Quignon, J., Allou, F., Absi, J., Petit, C. "Two-dimensional/three-dimensional biphasic modelling of the dynamic modulus of bituminous materials", European Journal of Environmental and Civil Engineering, 17(6), pp. 430-443, 2013. https://doi.org/10.1080/19648189.2013.786243

[17] Olard, F., Di Benedetto, H. "General "2S2P1D" Model and Relation Between the Linear Viscoelastic Behaviours of Bituminous Binders and Mixes", Road Materials and Pavement Design, 4(2), pp. 185224, 2003.

https://doi.org/10.1080/14680629.2003.9689946
[18] Cucalon, L. G., Rahmani, E., Little, D. N., Allen, D. H. "A multiscale model for predicting the viscoelastic properties of asphalt concrete", Mechanics of Time-Dependent Materials, 20(3), pp. 325-342, 2016. https://doi.org/10.1007/s11043-016-9303-2

[19] Cheng, Y., Tao, J., Jiao, Y., Tan, G., Guo, Q., Wang, S., Ni, P. "Influence of the properties of filler on high and medium temperature performances of asphalt mastic", Construction and Building Materials, 118, pp. 268-275, 2016. https://doi.org/10.1016/j.conbuildmat.2016.05.041

[20] Kim, J., Lee, H. S., Kim, N. "Determination of Shear and Bulk Moduli of Viscoelastic Solids from the Indirect Tension Creep Test", Journal of Engineering Mechanics, 136(9), pp. 1067-1075, 2010. https://doi.org/10.1061/(ASCE)EM.1943-7889.0000151

[21] Beskou, N. D., Hatzigeorgiou, G. D., Theodorakopoulos, D. "Dynamic inelastic analysis of 3-D flexible pavements under moving vehicles: A unified FEM treatment", Soil Dynamics and Earthquake Engineering, 90, pp. 420-431, 2016. https://doi.org/10.1016/j.soildyn.2016.09.018

[22] Tarasovs, S., Kruminš, J., Tamužs, V. "Modelling of the fracture toughness anisotropy in fiber reinforced concrete", Frattura Ed Integrita Strutturale, 10(35), pp. 271-277, 2016. https://doi.org/10.3221/IGF-ESIS.35.31

[23] Bocz, P. "The effect of stiffness and duration parameters to the service life of the pavement structure", Periodica Polytechnica Civil Engineering, 53(1), pp. 35-41, 2009. https://doi.org/10.3311/pp.ci.2009-1.05 